\title{
Adatom on Graphene, Directly Imaged by Aberration Corrected TEM at 300kV
}

\author{
T. Tanaka****, Y. Abe*, H. Sawada*****, E. Okunishi*****, Y. Kondo******, Y Tanishiro****, \\ K. Takayanagi $* * * *$
}

* Department of Physics, Tokyo Institute of Technology, 2-12-1-H-51, Oh-okayama, Meguro-ku, Tokyo 152-8551, Japan

** JEOL Ltd., 3-1-2 Musashino, Akishima, Tokyo 196-8558, Japan

*** Japan Science and Technology Agency, CREST, 5, Sanbancho, Chiyoda-ku, Tokyo 102-0075, Japan

A new spherical aberration (Cs) corrected TEM-STEM microscope with a cold field emission gun, called R005 microscope [1-5], has been used to test its performance in TEM studies on graphene sheets.

A thin film of graphite is cleaved from highly oriented pyrolytic graphite (HOPG) and is mounted to a double-tilt specimen holder of the R005 microscope. Neither glues nor solvents have been used during the specimen preparation processes. A graphene sheet attached at an edge of the thin graphite crystal is observed at focus steps of $1 \mathrm{~nm}$, where the spherical aberration Cs is tuned to zero using Diffractogram tableau method [5].

Figure 1 shows a high-resolution TEM image of a graphene sheet. As shown in Fig.1, the TEM images taken in a preferred defocus condition show the honeycomb structure, which consists of bright dots. The bright dots are analyzed to have a unit intensity, where the intensity is measured referring to the intensity of the primary electron beam. The intensity of the dots has some noises around $10 \%$ of the unit intensity, except some dots having twice of the unit intensity. Therefore, the bright dots having the unit intensity are confirmed as images of single carbon atoms. Image simulation by the multi-slice calculation showed quantitative agreement on the unit intensity with the experiment. The calculation was done using the imaging condition of $C s=0 \mathrm{~mm}, C c=1.5 \mathrm{~mm}$, energy spread of imaging electron $\Delta E=0.4 \mathrm{eV}$ at $300 \mathrm{kV}$ acceleration and beam convergence of $1 \mathrm{mrad}$. From several TEM images of different graphene sheets, defect structures have been found to be created in the sheets as shown in Fig. 2 (a-c); adatom at the bridge site, single vacancy, and double vacancies. These defects have been predicted theoretically [6] and reported in experiments [7-11]. Direct imaging of the adatom at the bridge site has never been done, since the projected distance between the adatom and the carbon atoms.

The TEM-EELS study in graphene will be reported in the poster session [12].

[1] F. Hosokawa et al., Proc. The 16th International Microscopy Congress, 2006, 582.

[2] H. Sawada et al., Japanese Journal of Applied Physics, 46 (2007) L568.

[3] H. Sawada et al., Microsc. Microanal. 14 (Suppl. 2), 2008, 802.

[4] T. Tomita et al., Microsc. Microanal. Proceeding, 2009.

[5] H. Sawada et al., Microsc Microanal 13 (Suppl 2), 2007, 880CD.

[6] A. J. Stones et al. J. Chem. Phys. Lett. 128 (1986) 501.

[7] J. C. Meyer et al., Nano Letters 8 (2008) 3582.

[8] J. C. Meyer et al., Nature 454 (2008) 319.

[9] H. Hashimoto et al., Nature 430 (2004) 870-873.

[10] K. Suenaga et al., Nature Nanotechnology 2 (2007) 358.

[11] M.H. Gass et al., Nature Nanotechnology, 3 (2008) 676. 
[12] Y. Abe, T. Tanaka et al., Microsc. Microanal. Proceeding, 2009.

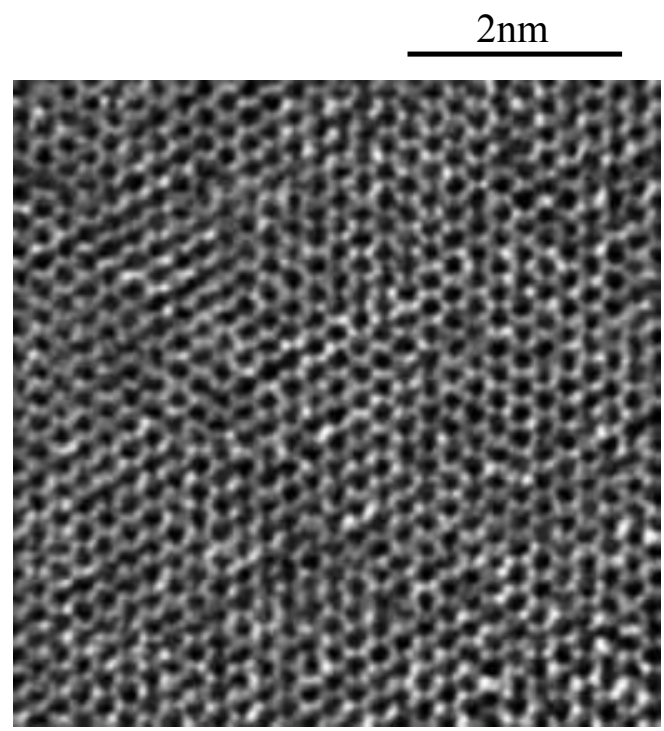

Fig. 1 Direct image of graphene. A bright dot was analyzed to be an image of single-carbon atom as described in the text.

(a)
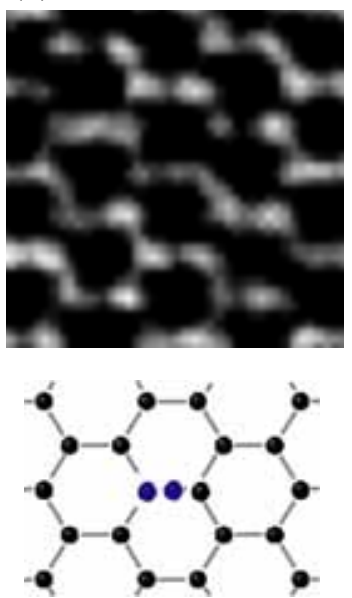

(b)
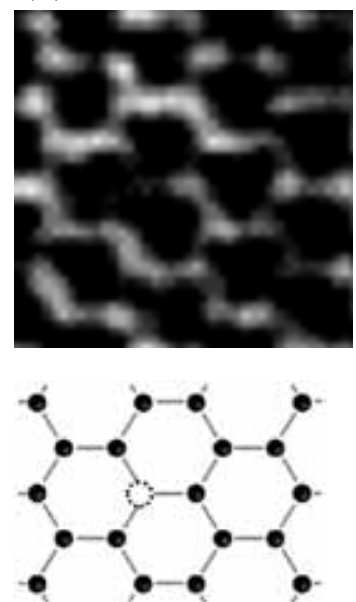

(c)
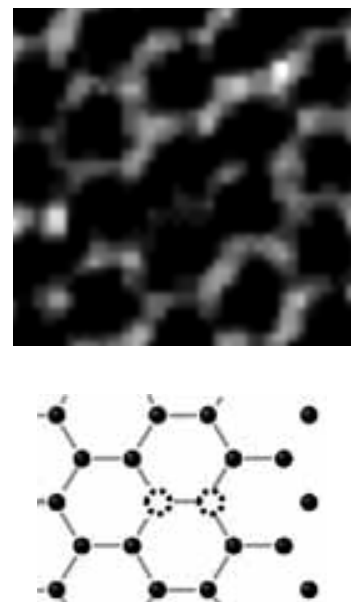

Fig.2 TEM images and the corresponding models of the various defects, (a) adatom at bridge site, (b) single vacancy, (c) double vacancy. 